\title{
A. NEGRO \\ M. MilanesE \\ Contrôle de min-max et feedback linéaire pour des systèmes dynamiques approchés en norme
}

Revue française d'automatique, informatique, recherche opérationnelle. Analyse numérique, tome 10, nº 1 (1976), p. 61-80.

$<$ http://www.numdam.org/item?id=M2AN_1976_10_1_61_0>

(C) AFCET, 1976, tous droits réservés.

L'accès aux archives de la revue "Revue française d'automatique, informatique, recherche opérationnelle. Analyse numérique » implique l'accord avec les conditions générales d'utilisation (http://www.numdam.org/legal. php). Toute utilisation commerciale ou impression systématique est constitutive d'une infraction pénale. Toute copie ou impression de ce fichier doit contenir la présente mention de copyright.

\section{Numdam}

Article numérisé dans le cadre du programme

Numérisation de documents anciens mathématiques

http://www.numdam.org/ 
R A I R O

ANALYSE NUMÉRIQUE

(Vol 10, n 3, mars 1976, p 61 a 82)

\title{
CONTROLE DE MIN-MAX ET FEEDBACK LINEAIRE POUR DES SYSTEMES DYNAMIQUES APPROCHES EN NORME $(*)$
}

\author{
par A. Negro $\left({ }^{1}\right)$ et M. Milanese $\left({ }^{2}\right)$ \\ Communiqué par P -J LAURENT
}

\begin{abstract}
Résumé - On consıdère le problème du feed-back linéaire pour un système dynamıque décrıt par un modèle gouverne par une équatıon d'évolution abstratte L'approximation entre système et modèle etant donnee par la norme de la différence des deux opérateurs d'évolution, on se redult à un problème de min-max avec contraintes, nonusuel

On résoud ce problème en boucle ouverte et en boucle fermée et on donne des condittons pour l'équivalence des deux contrôles
\end{abstract}

\section{INTRODUCTION}

On considère un problème usuel de contrôle optimal, avec une fonction coût quadratique, pour un système décrit au moyen d'un modèle linéaire gouverné par une équation d'évolution abstraite.

Une mesure convenable de l'approximation entre le système et le modèle est donnée par la norme de la différence des deux opérateurs d'évolution, qu'on peut estimer par les méthodes de Donat1-Milanese [3], Milanese [10], Milanese-Negro [11], Negro [12].

Utilisant cette estımation on se réduit à un problème de mın-max pour le modèle avec la sortie modifiée par une erreur bornée en fonction de la commande (Menga-Milanese [9]).

(*) Cette etude a été effectuée avec l'aide financiere du C N R Italien

(1) A Negro Istıtuto dı Analisı Matematica, Universita dı Torino e Gruppo di Informatica e Automatica, Istituto Elettrotecnico Nazıonale Gahleo Ferrarıs-Politecnico di Torıno

(2) M Milanese Gruppo di Informatica e Automatica, Istituto Elettrotecnico Nazionale Galıleo Ferrarıs-Politecnico di Torıno

Revue Françaıse d'Automatıque, Informatıque et Recherche Opératıonnelle ${ }^{\circ}$ mars 1976 
Ce problème de min-max n'est pas usuel dans la mesure où l'ensemble, sur lequel la maximisation porte, dépend de la variable qui tend à minimiser. On peut quand même le résoudre et caractériser la solution en boucle ouverte.

On se pose alors le problème du feedback linéaire sur la sortie du système : on démontre que, si une condition supplémentaire sur le modèle n'est pas satisfaite, le coût optimal en boucle fermée est supérieur au coût en boucle ouverte.

Dans la démonstration on utilise des résultats sur les opérateurs de Riccati analogues à ceux qu'on peut trouver dans Faurre [5].

\section{DEFINITION DE L'INCERTITUDE ET FORMULATION DU PROBLEME DE CONTROLE}

On considère un problème de contrôle optimal pour un système dynamique linéaire $S$, sur l'intervalle de temps $[O, T]$ avec $T \leqslant+\infty$.

Soient $U$ et $Y$ deux espaces de Hilbert séparables. Si $u_{p} \in L^{2}(-\infty, O ; U)$ est la commande passée et $u \in L^{2}(O, T ; U) \equiv \mathcal{U}$ est le contrôle présent, alors la sortie du système $y_{s} \in L^{2}(O, T ; Y) \equiv \mathrm{Y}$ peut s'écrire sous la forme

$$
y_{s}=\Lambda_{s} u_{p}+W_{s} u
$$

et l'on supposera que $\Lambda_{s}, W_{s}$ soient des opérateurs (linéaires) bornés.

Le problème usuel du régulateur pour le système $S$ est le suivant :

Problème 1. - Trouver $u \in U$ tel que

$$
J_{s}(u) \leqslant J_{s}(v) \quad \forall v \in \mathcal{U}
$$

ou

$$
J_{s}(u)=\|u\|_{\text {U }}^{2}+\left\|y_{s}\right\|_{y}^{2}=\|u\|_{\text {U }}^{2}+\left\|\Lambda_{s} u_{p}+W_{s} u\right\|_{y}^{2}
$$

Toutefois dans ce travail on veut considérer le cas dans lequel les équations d'état de $S$ ne sont pas connues et toute l'information sur les opérateurs $\Lambda_{s}$ et $W_{s}$ est donnée par un ensemble de mesures d'entrée-sortie.

Utilisant les méthodes d'« approximation en norme » (Donati-Milanese [3], Milanese [10], Milanese-Negro [11], Negro [12]) on peut décrire $S$ comme la somme d'un modèle linéaire $M$ (qui peut être optimal dans une classe donnée) et d'un système erreur $\varepsilon$ pour lequel on connaît une estimation de la norme de l'opérateur d'évolution.

Pour décrire le modèle $M$ on fera les hypothèses suivantes : soient $V^{\prime}$ et $H$ des espaces de Hilbert séparables, $V \subset H$ avec injection continue, $V$ dense dans $H$. Lorsque $H$ est identifié à son dual on a $H \subset V^{\prime}$ avec injection continue. Soit $A \in \mathfrak{L}\left(V, V^{\prime}\right)$ tel que

$$
-(A v, v)_{V^{\prime}, V} \geqslant a\|v\|_{V}^{2}, a>0, \quad \forall v \in V .
$$


Soient $B \in \mathfrak{L}(U, H), C \in \mathfrak{L}(V, Y)$; l'état $x(t)$ et la sortie $y(t)$ du modèle $M$ sont donnés par les équations

$$
\begin{aligned}
& \left.\frac{\mathrm{d} x}{\mathrm{~d} t}(t)=A x(t)+B u(t) \quad t \in\right] 0, T[, \\
& x(0)=x_{0}=\Pi u_{p} \in H, \\
& y(t)=C x(t)
\end{aligned}
$$

Il est bien connu (Lions [8]) que (5), (6) ont une solution unique quelque soit $u \in U$ et que la solution est continue à valeurs dans $H$. La donnée initiale $x_{0}$ est une fonction $(\Pi)$ bornée de la commande passée du système.

La sortie $y$ du modèle est fonction affine de $u$ :

$$
y=W u+f
$$

où

$$
\begin{aligned}
& (W u)(t)=\int_{0}^{t} C \mathrm{e}^{(t-s) A} B u(s) \mathrm{d} s, \\
& f(t)=C \mathrm{e}^{t A} x_{0}=C \mathrm{e}^{t A} \Pi u_{p} .
\end{aligned}
$$

Remarque. - Peut être parfois commode de supposer que l'image de $\Pi$ est même dans $D(A)$, ce que n'est pas très restrictif puisque il s'agit d'un modèle. alors

Soit $e$ la sortie du système erreur $\varepsilon$, correspondante au contrôle $u$; on a

$$
y_{s}(t)=y(t)+e(t) \quad t \in[O, T] .
$$

Les estimations sur la norme de l'opérateur d'évolution de $\varepsilon$ donnent la contrainte

$$
e \in \Sigma(u)=\left\{e \in \mathcal{Y} \mid\|e\|_{\mathcal{Y}}^{2} \leqslant E^{2}\|u\|_{\mathscr{u}}^{\dot{2}}+\omega^{2},\right.
$$

où les constantes $E^{2}$ et $\omega^{2}$ dépendent seulement de l'ensemble des mesures d'entrée-sortie utilisé dans l'approximation en norme et de la commande passée $u_{p}$.

On définit

$$
J(u, e)=\|u\|_{u}^{2}+\|W u+f+e\|_{y}^{2}
$$

(avec $f \in \mathcal{Y}$ et $W: \mathcal{U} \rightarrow \mathcal{y}$ ).

On envisage une solution de min-max pour le problème $1: \Sigma(u)$ étant la boule fermée de centre $O$ et de rayon $b(u)=\sqrt{E^{2}\|u\|_{u}^{2}+\omega^{2}}$ dans $Y$, on forme

$$
I(u)=\max _{e \in \Sigma(u)} J(u, e)
$$

$\mathrm{n}^{\circ}$ mars 1976. 
et l'on cherche $u^{*}$ tel que

$$
I\left(u^{*}\right)=\min _{u \in \Upsilon} I(u)
$$

Donc $u^{*}$ est solution du

Problème 2. - Trouver $u^{*} \in U$ tel que

$$
I\left(u^{*}\right)=\min _{u \in \mathcal{U}} \max _{e \in \Sigma(u)} J(u, e) .
$$

Pour chaque $u$ donné, le problème (14) est simple puisqu'il s'agit de trouver le point $\psi(u)$ de $\Sigma(u)$ à distance maximum de l'élément $-W u-f$ :

$$
a(u)=\|W u+f\|
$$

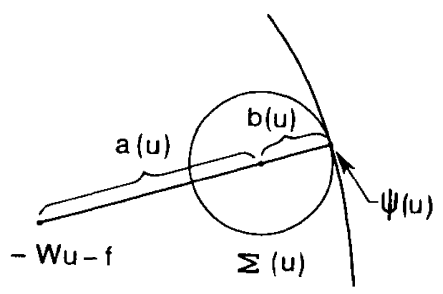

Figure 0

On a : $\psi(u)=\alpha(W u+f)$ avec $\alpha$ tel que $\psi(u)$ soit sur la frontière de $\Sigma(u)$

Il est alors clair que $I(u)=\|u\|^{2}+(a(u)+b(u))^{2}$.

$\mathrm{Au}$ numéro suivant on démontre brièvement l'existence et l'unicité de la solution de (15).

On a donc une solution du problème 2 en boucle ouverte. Mais le but essentiel de ce travail est de vérifier si le contrôle optimal peut être synthétisé, par analogie au cas classique, au moyen d'un feedback linéaire sur l'état du système.

Pour cela on aura besoin de la caractérisation suivante (numéro 3 ) de $u^{*}$ : la contrainte $e \in \Sigma(u)$ s'écrit $g(u, e) \leqslant 0$ avec $g(u, e)=\|e\|^{2}-E^{2}\|u\|^{2}-\omega^{2}$.

On introduit alors :

$$
J_{\gamma}(u, e)=J(u, e)-\gamma g(u, e)
$$

qui, pour $\gamma>1$, est convexe-concave et a un point de selle unique. On montre qu'il existe $\gamma^{*}$ unique pour lequel le point de selle correspondant $\left[u^{*}, e^{*}\right]$ est tel que $g\left(u^{*}, e^{*}\right)=0$. Ce point de selle est alors solution de (14) et (15) et l'on a :

$$
\gamma^{*}=\frac{b\left(u^{*}\right)+a\left(u^{*}\right)}{b\left(u^{*}\right)}
$$


On forme alors (numéro 4) :

$$
\begin{aligned}
I^{*}(u) & =\left(1+\gamma^{*} E^{2}\right)\|u\|^{2}+\frac{\gamma^{*}}{\gamma^{*}-1}\|W u+f\|^{2}= \\
& =\max _{e \in \Sigma(u)} J_{\gamma^{*}}(u, e)-\gamma^{*} \omega^{2}=I(u)-\gamma^{*} \omega^{2} .
\end{aligned}
$$

Le problème 2 est donc équivalent au calcul de $\gamma^{*}$ et au problème quadratique :

$$
I^{*}\left(u^{*}\right)=\min _{u \in \mathcal{u}} I^{*}(u)
$$

qui, moyennant l'équation de Riccati associée, donne $u^{*}$ comme feedback linéaire sur l'état du modèle :

$$
u^{*}(t)=L^{*} x^{*}(t) .
$$

Avec une condition supplémentaire sur le choix du modèle, on verra dans la suite que l'on peut modifier l'opérateur $L^{*}$ de façon à obtenir un feedback linéaire optimal sur l'état du système (numéros 4 et 5).

Il est possible de rẻduire le problème 2 à la recherche des points de selle de la fonction (17) si la méthode classique des multiplicateurs de Lagrange est valable dans les hypothèses sous lesquelles le problème est donné.

Or il est connu que, même en dimension finie (Danskin [2]), l'utilisation de la méthode des multiplicateurs de Lagrange dans les problèmes de min-max est subordonnée, bien entendu à des questions de régularité, mais aussi à l'unicité du maximum pour toute valeur fixée de la variable minimisante.

Donc les résultats du numéro 3 sont simples, mais non triviales, comme il est montré par l'exemple suivant (fig. 1).

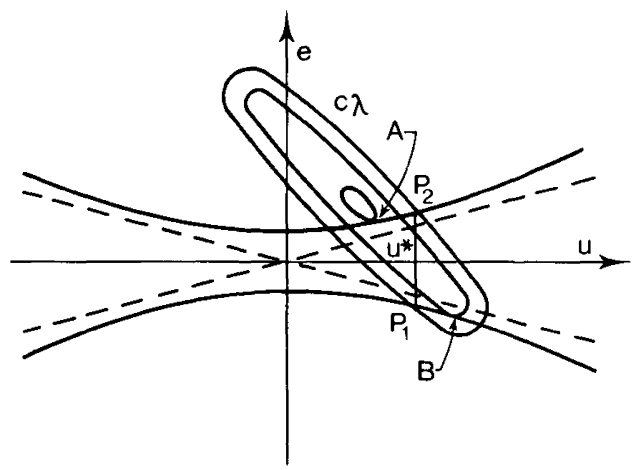

Figure 1

Les ellipses $c_{\lambda}$ sont les lignes de niveau de

$$
\begin{aligned}
J(u, e)=[(u-p) \cos \alpha-(e-q) \sin \alpha]^{2} & \\
& +\beta^{-2}[(u-p) \sin \alpha+(e-q) \cos \alpha]^{2},
\end{aligned}
$$

$\mathrm{n}^{\circ}$ mars 1976. 
avec $p, q, \alpha, \beta$ convenables; l'hyperbole $\Gamma$ est le bord de l'ensemble $\left\{[u, e] \in \mathbb{R}^{2} / e \in \Sigma(u)\right\}$ défini par

$$
g(u, e)=e^{2}-E^{2} u^{2}-\omega^{2} \leqslant 0 .
$$

Dans la figure 1 la ligne en gras donne les points $(u, \bar{e})$ tels que

$$
J(u, \bar{e})=\max _{g(u, e) \leqslant 0} J(u, e) .
$$

Pour $u=u^{*}$ la ligne est discontinue (il y a deux points $P_{1}$ et $P_{2}$ de maximum) et pourtant $u^{*}$ réalise le min-max de $J$ sous la contrainte $g \leqslant 0$.

La méthode des multiplicateurs de Lagrange donne seulement les points $A$ et $B$ qui ne sont pas de min-max.

\section{EXISTENCE ET UNICITE DU CONTROLE DE MIN-MAX}

Lemme 1. - Si $x_{0} \neq 0$, quelque soit $u \in \mathcal{U}$ il y a une solution unique $\psi(u)$ de l'équation

$$
J\left(u, \psi(u)=\max _{e \in \Sigma(u)} J(u, e),\right.
$$

qui est donnée par

$$
\psi(u)=\alpha x=\alpha(W u+f), \quad \alpha=\frac{\sqrt{E^{2}\|u\|^{2}+\omega^{2}}}{\|W u+f\|} .
$$

En tout cas

$$
I(u)=\max _{e \in \Sigma(u)} J(u, e)=\|u\|^{2}+\left\{\|W u+f\|+\sqrt{E^{2}\|u\|^{2}+\omega^{2}}\right\}^{2} .
$$

Démonstration. - On remarque que si $\mathrm{u}_{0} \neq 0, \forall u \in \mathcal{u}$ on a $W u+f \neq 0$ et alors évidemment le maximum de $\|W u+f+e\|^{2}$ dans la boule de centre $O$ et de rayon $\left[E^{2}\|u\|^{2}+\omega^{2}\right]^{1 / 2}$ est atteint en $\psi(u)$. Si $W u+f=0$ on a quand même $I(u)=\|u\|^{2}+E^{2}\|u\|^{2}+\omega^{2}$.

c.q.f.d.

Lemme 2. - $I(u)$ est strictement convexe et continue sur $\mathcal{u}$.

Démonstration. - Il est bien connu que si $f$ est convexe et monotone non-décroissante et $g$ est convexe, alors $f \circ g$ est convexe.

Mais les fonctions $z \rightarrow \sqrt{E^{2} z^{2}+\omega^{2}}$ et $z \rightarrow z^{2}$ sont convexes et croissantes pour $z \geqslant 0$ et $\|\cdot\|$ est aussi convexe; donc $u \rightarrow\left\{\|W u+f\|+E^{2}\|u\|^{2}+\omega^{2}\right\}^{2}$ est convexe. Puisque $u$ est un espace de Hilbert, $u \rightarrow\|u\|^{2}$ est strictement convexe.

La continuité de $I$ est triviale. 
Théorème 1. - Le problème $2 \mathrm{a}$ une solution unique.

Démonstration. - On peut présenter le problème 2 sous la forme : trouver $u \in \mathcal{U}$ tel que $I(u) \leqslant I(v), \forall v \in \mathcal{U}$. Mais I est convexe et continue, donc s.c.i. (semi continue inférieurement) pour la topologie faible de $U$. On peut vérifier immédiatement que $I(u) \rightarrow+\infty$ et alors par un théorème bien connu on a l'existence du minimum.

La convexité stricte implique l'unicité.

c.q.f.d.

\section{LA METHODE DES MULTIPLICATEURS DE LAGRANGE}

On considère la fonction

$$
\begin{aligned}
J_{\gamma}(u, e) & =J(u, e)-\gamma\left[\|e\|^{2}-E^{2}\|u\|^{2}-\omega^{2}\right] \\
& =\|u\|^{2}+\|W u+f+e\|^{2}-\gamma\left[\|e\|^{2}-E^{2}\|u\|^{2}-\omega^{2}\right],
\end{aligned}
$$

avec $\gamma \in \mathbb{R}$.

Proposition 1. - Quel que soit $\gamma>1$ il existe un point de selle unique $\left(u_{\gamma}, e_{\gamma}\right)$ de $J_{\gamma}$ sur $\mathcal{U} \times \mathbf{y}$, c'est-à-dire

$$
J_{\gamma}\left(u_{\gamma}, e\right) \leqslant J_{\gamma}\left(u_{\gamma}, e_{\gamma}\right) \leqslant J_{\gamma}\left(u, e_{\gamma}\right) \quad \forall e \in \mathcal{Y}, \quad \forall u \in \mathcal{U} .
$$

Le point de selle est la solution unique du système

$$
D_{e} J_{\gamma}=0, \quad D_{u} J_{\gamma}=0,
$$

où

$$
\begin{aligned}
& D_{e} J_{\gamma}=W u+f+e-\gamma e, \\
& D_{u} J_{\gamma}=u+W^{*}(W u+f+e)+\gamma E^{2} u
\end{aligned}
$$

sont les dérivées de Fréchet de $J_{\gamma}$ par rapport à $e$ et $u$.

Démonstration. $-J_{\gamma}(\cdot, e)$ est strictement convexe, s.c.i. pour la topologie faible de $u$ et $J_{\gamma}(u, e) \rightarrow+\infty$ lorsque $\|u\| \rightarrow+\infty$. Si $\gamma>1, J_{\gamma}(u, \cdot)$ est strictement concave, s.c.s. (semi continue supérieurement) pour la topologie faible de $\mathcal{Y}$ et $J_{\gamma}(u, e) \rightarrow-\infty$ lorsque $\|e\| \rightarrow+\infty$. Puisque $\mathcal{U}$ et $\boldsymbol{y}$ sont des espaces de Hilbert, un théorème bien connu (Bensoussan [1], Lemaire [7], Ekeland-Temam [4]) donne l'existence et l'unicité du point de selle.

Mais $J_{\gamma}$ est Fréchet-différentiable et alors le point de selle est caractérisé $\operatorname{par}(25)$.

$$
\text { c.q.f.d. }
$$


Théorème 2. - Si $x_{0} \neq 0$ et si $\omega^{2} \neq 0$, le système d'équations

$$
\begin{gathered}
W u+f+(1-\gamma) e=0 \\
\left(1+\gamma E^{2}\right) u+W^{*}(W u+f+e)=0 \\
\|e\|^{2}-E^{2}\|u\|^{2}-\omega^{2}=0
\end{gathered}
$$

a une solution unıque $\left.\left(u^{*}, e^{*}, \gamma^{*}\right) u \times y \times\right] 1,+\infty\left[\right.$. De plus $\left(u^{*}, e^{*}\right)$ réalise le min-max de $J$ :

$$
J\left(u^{*}, e^{*}\right)=\min _{u \in \mathcal{U}} \max _{e \in \Sigma(u)} J(u, e)
$$

et $\gamma^{*}=\frac{\alpha^{*}+1}{\alpha^{*}}$ où

$$
\alpha^{*}=\frac{\sqrt{E^{2}\left\|u^{*}\right\|^{2}+\omega^{2}}}{\left\|W u^{*}+f\right\|} .
$$

Remarque. - Par la proposition 1, pour chaque $\gamma>1$ il y a une solution unique de (28), (29). Ce n'est pas évident à priori l'unicité de $\gamma \in] 1,+\infty[$ tel que le point de selle correspondant satisfait à la contrainte (30).

Démonstration. - Pour l'unicité on remarque que $\forall_{\gamma}$ on a

$$
J_{\gamma}(u, \psi(u))=J(u, \psi(u))=I(u)
$$

Par (28) $e^{*}=\left(\gamma^{*}-1\right)^{-1}\left(W u^{*}+f\right)$ et par (30), puisque $\gamma^{*}-1>0$, on a $\left(\gamma^{*}-1\right)^{-1}=\alpha^{*}$. Donc $e^{*}=\psi\left(u^{*}\right)$.

Il suffit de démontrer que $I(u)$ est minimum en $u^{*}$, parce que $I$ est strictement convexe. Mais dans les hypothèses du théorème $\psi$ n'est pas seulement parfaitement définie, mais aussi Fréchet-différentiable.

Donc

$$
D_{u} I(u)=D_{u} J_{\gamma^{*}}(u, \psi(u))+D_{e} J_{\gamma^{*}}(u, \psi(u)) . D_{u} \psi(u)
$$

et comme $D_{u^{*}} J_{\gamma^{*}}=D_{e} J_{\gamma^{*}}=0$ en $\left(u^{*}, \psi\left(u^{*}\right)\right), I\left(u^{*}\right)$ est minimum.

L'existence est une conséquence immédiate de la théorie usuelle des multiplicateurs de Lagrange (Schwartz [13]) lorsque on remarque que :

$$
\min _{u \in \mathcal{U}} \max _{e \in \Sigma(u)} J(u, e)=\min _{u \in \mathcal{U}} \max _{e \in \bar{\Sigma} \Sigma(u)} J(u, e),
$$

où

$$
\partial \Sigma(u)=\left\{e \in \mathcal{Y}\|e\|^{2}-E^{2}\|u\|^{2}-\omega^{2}=0\right\}
$$


De toute façon si $\left(u^{*}, e^{*}\right)$ est le point de min-max de $J$, dont l'existence (et l'unicité) a été démontrée au Théorème 1 , et si $\gamma^{*}=\frac{\alpha^{*}+1}{\alpha^{*}}$, alors

$$
\begin{aligned}
& \frac{1}{2} D_{u} I\left(u^{*}\right)=\left(1+\gamma^{*} E^{2}\right) u^{*}+\left(1+\alpha^{*}\right) W^{*}\left(W u^{*}+f\right)=0, \\
& e^{*}=\psi\left(u^{*}\right)=\left(\gamma^{*}-1\right)^{-1}\left(W u^{*}+f\right)
\end{aligned}
$$

et $\left(u^{*}, e^{*}, \gamma^{*}\right)$ satisfont (28), (29), (30) c.q.f.d.

Remarque. - On peut souligner que les résultats de ce numéro sont liés à la structure particulière de $I$ et $g$ pour l'unicité de $\gamma^{*}$ dans $] 1,+\infty[;$ au contraire l'existence des multiplicateurs de Lagrange dépend de l'unicité du maximum et pas de la structure quadratique de $I$ et $g$.

\section{BOUCLE OUVERTE ET BOUCLE FERMEE}

Par le théorème 2 , le problème 2 se réduit au

Problème 3. - Trouver $u^{*} \in \mathcal{U}$ tel que

$$
I^{*}\left(u^{*}\right) \leqslant I^{*}(v) \quad \forall v \in \mathcal{U},
$$

où

$$
I^{*}(u)=\left(1+\gamma^{*} E^{2}\right)\|u\|^{2}+\frac{\gamma^{*}}{\gamma^{*}-1}\|y\|^{2}
$$

avec

$$
\frac{\mathrm{d} x}{\mathrm{~d} t}=A x+B u, \quad x(0)=x_{0}, \quad y=C x .
$$

En effet $I^{*}(u)+\gamma^{*} \omega^{2}=J_{\gamma^{*}}(u, \psi(u))=J(u, \psi(u))=I(u)$.

Alors il est bien connu que le contrôle optimal $u^{*}(t)$ peut être réalisé comme fonction linéaire de l'état $x(t)$ au même instant.

$$
u^{*}(t)=-\frac{1}{1+\gamma^{*} E^{2}} B^{*} P_{\gamma^{*}}(t) x(t)
$$

où $P_{\gamma^{*}}(t) \in \mathfrak{L}(H), P_{\gamma^{*}}^{*}(t)=P_{\gamma^{*}}(t),\left(P_{\gamma^{*}}(t) h, h\right) \geqslant 0 \quad \forall h \in H$ et $P_{\gamma^{*}}$ satisfait à une équation de Riccati (Lions [8]).

Dans la suite on considèrera le cas $T=+\infty$ de façon que $P_{\gamma^{*}}(t)=P_{\gamma^{*}}$ indépendant de $t$, par le principe d'optimalité, et l'on écrira

$$
u^{*}(t)=L^{*} x(t) \text {. }
$$

$n^{\circ}$ mars 1976. 
La situation est montrée dans la figure 2 a où $e$ est maintenant une sortie admissible quelconque du système erreur $\varepsilon$ et pas nécessairement la pire. On a fait $y=x$, c'est-à-dire $Y=H$ et $C=I$.

Cette hypothèse est nécessaire dans la suite.

Évidemment $L^{*}$ ne donne pas une solution en boucle fermée du problème de contrôle parce que $L^{*}$ est un feedback sur la sortie du modèle et pas sur la sortie du système.

Un feedback sur la sortie du système est montré dans la figure $2 \mathrm{~b}$, où $L$ est un élément quelconque de $\mathfrak{L}(H, U)$.

Alors on considère le problème suivant : soit

$$
x=X(e, \tilde{L}), e \in \mathcal{Y}, \tilde{L} \in \mathfrak{L}(H, U)
$$

la solution unique dans $L_{\mathrm{loc}}^{2}(0,+\infty ; V)$ de

$$
\frac{\mathrm{d} x}{\mathrm{~d} t}=A \dot{x}+B \tilde{L}(x+e), \quad x(0)=x_{0} .
$$

L'existence et l'unicité de $x$ sont conséquences immédiates du fait que $A$ est générateur infinitésimal d'un semi-groupe de contractions et que $B \tilde{L}$ est borné de $H$ dans $H$. On sait de plus que $\mathrm{d} x / \mathrm{d} t \in L_{\mathrm{loc}}^{2}\left(0,+\infty ; V^{\prime}\right)$ et que $x$ est continu à valeurs dans $H$.

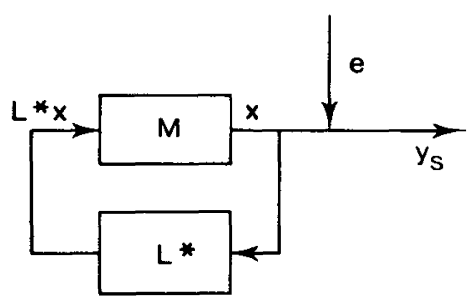

Figure 2 a

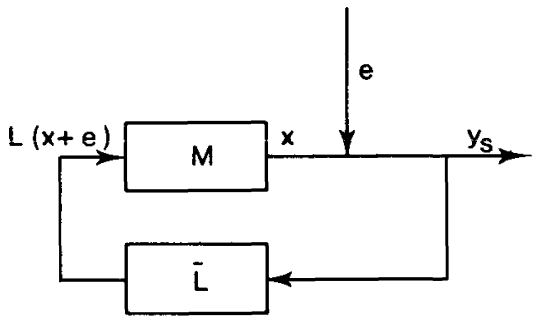

Figure 2 b

On définit alors la fonction $K: \mathfrak{L}(H, U) \times \boldsymbol{y} \rightarrow[0,+\infty]$ par

$$
K(\tilde{L}, e)=\|\tilde{L}(X(e, \tilde{L})+e)\|_{u}^{2}+\|X(e, \tilde{L})+e\|_{y}^{2}
$$

(on prend $K=+\infty$ si $x \notin y$ ).

On définit également l'ensemble

$$
\Gamma(\tilde{L})=\left\{e \in \mathcal{Y} \mid\|e\|_{y}^{2} \leqslant E^{2} \| \tilde{L}\left(X(e, \tilde{L})+e \|_{\dddot{q}}^{2}+\omega^{2}\right\}\right.
$$

$($ Si $\tilde{L}(X(e, \tilde{L})+e) \notin$ u on a $e \in \Gamma(\tilde{L}))$. 
Avec ces définitions on considère le

Problème 4. - Trouver $\tilde{L}^{*} \in \mathfrak{L}(H, U)$ telle que

$$
\sup _{e \in \Gamma\left(\tilde{L}^{*}\right)} K\left(\tilde{L}^{*}, e\right)=\min _{\tilde{L} \in \mathcal{L}(H, U)} \sup _{e \in \Gamma(\tilde{L})} K(\tilde{L}, e)
$$

Un premier résultat relativement à ce problème est donné par le

Théorème 3. - Sous les hypothèses $x_{0} \in D(A), x_{0} \neq 0$ et $\omega^{2} \neq 0$, on a l'inégalité :

$$
\inf _{\tilde{L} \in \tilde{L}(H, U)} \sup _{e \in \Gamma(\tilde{L})} K(\tilde{L} e) \geqslant \min _{u \in \mathcal{U}} \max _{e \in \Sigma(u)} J(u, e) .
$$

On verra plus loin que, avec des hypothèses convenables sur le modèle $M$, il y a égalité en (48).

Démonstration. - Soit $\mathcal{M}$ le sous-ensemble de $\mathcal{L}(H, U)$ formé des $L$ telles que la solution $x(L)$ de

$$
\frac{\mathrm{d} x(L)}{\mathrm{d} t}=(A+B L) x(L), x(L)(0)=x_{0}
$$

appartient à Y (fig. 2 a).

On définit alors $\emptyset: \mathcal{M} \times \mathcal{Y} \rightarrow[0,+\infty[$ par

$$
\emptyset(L, e)=J(L x(L), e)
$$

Évidemment on a

$$
\min _{L \in \mathcal{H}} \max _{e \in \Sigma(L x(L))} \emptyset(L, e)=\emptyset\left(L^{*}, e^{*}\right)=\min _{u \in \mathcal{U}} \max _{e \in \Sigma(u)} J(u, e),
$$

où $L^{*}$ est défini par (41), (42) et $e^{*}=\alpha\left(L^{*}\right) x\left(L^{*}\right)$, avec

$$
\alpha(L)=\frac{\sqrt{E^{2}\|L x(L)\|^{2}+\omega^{2}}}{\|x(L)\|}
$$

Remarque. - Si on considère la fonction $\mu(L)=\frac{1}{1+\alpha(L)} L$ définie de $\mathcal{M}$ dans $\mathcal{L}(H, U)$, on a

$$
\begin{aligned}
\sup _{e \in \Gamma(\mu(L))} K(\mu(L), e) \geqslant K(\mu(L), \alpha(L) x(L)) & \\
& =\emptyset(L, \alpha(L) x(L))=\max _{e \in \Sigma(L x(L))} \emptyset(L, e) .
\end{aligned}
$$

En effet si $e=\alpha(L) x(L)$, alors $x(L)$ est solution de

$$
\mathrm{d} x / \mathrm{d} t=A x+B \mu(L)(x+e), \quad x(0)=x_{0}
$$

$\mathrm{n}^{\circ}$ mars 1976. 
et donc $X(e, \mu(L))=x(L)$. De plus

$$
\alpha(L) x(L) \in \Sigma(L x(L))=\Sigma(\mu(L)(x(L)+\alpha(L) x(L)))
$$

et alors $\alpha(L) x(L) \in \Gamma(\mu(L))$.

Maintenant il suffirait de montrer que l'infimum en (48) est égal à l'infimum sur l'image de $\mu$. On n'a pas exactement ça, mais on peut démontrer les propositions suivantes.

Proposition 2. - Soit $E\|\tilde{L}\| \geqslant 1$, alors $\forall M>0$ on peut trouver $e \in \mathcal{Y}$ telle que $\|e\|_{y} \geqslant M$ et $e \in \Gamma(\tilde{L})$.

On a donc

$$
\sup _{e \in \Gamma(\tilde{L})} K(\tilde{L}, e)=+\infty
$$

Proposition 3. - Soit $E\|\tilde{L}\|<1$. On définit

$$
\begin{gathered}
\alpha_{T}(L)=\frac{\sqrt{E^{2}\|L x(L)\|_{T}^{2}+\omega^{2}}}{\|x(L)\|_{T}}\left({ }^{1}\right) \\
\mu_{T}(L)=\left(1+\alpha_{T}(L)\right)^{-1} L, \quad T<+\infty .
\end{gathered}
$$

Alors $\forall T \in] 0,+\infty[$ il existe $L \in \mathfrak{L}(H, U)$, dépendant de $T$, tel que $\tilde{L}=\mu_{T}(L)$, c'est-à-dire

$$
\{\tilde{L} \in \mathfrak{L}(H, U) \mid E\|\tilde{L}\|<1\} \subset \operatorname{Im}_{T} \quad \forall T<+\infty
$$

Démonstration de la Proposition 2. - Pour tout $\varepsilon>0$ il existe $h \in H$ tel que $\|h\|=1$ et $\|\tilde{L} h\| \geqslant\|\tilde{L}\|-\varepsilon$. Soit $e_{n} \in \mathcal{Y}$ défini par

$$
e_{n}(t)=\left\{\begin{array}{lll}
z \sqrt{n} h & \text { lorsque } & t \in\left[0, \frac{1}{n}\right] \\
0 & \text { lorsque } & t>n^{-1},
\end{array}\right.
$$

alors $\left\|e_{n}\right\|_{y}^{2}=z^{2}$ et la solution de l'équation $\frac{\mathrm{d} x}{\mathrm{~d} t}=A x+B L\left(x+e_{n}\right), x(0)=\mathrm{u}_{0}$ est donnée par

$$
x(t)=\mathrm{e}^{(A+B \tilde{L}) t} x_{0}+\int_{0}^{T} \mathrm{e}^{(A+B \tilde{L})(t-s)} B \tilde{L} z \sqrt{n} h \mathrm{~d} s
$$

(1) Si $f \in L_{\text {loc }}^{2}(0,+\infty ; B)$ avec $B$ espace de Banach, on note

$$
\|f\|_{T}^{2}=\int_{0}^{T}\|f(t)\|_{B}^{2} \mathrm{~d} t .
$$


pour $t \in\left[0, n^{1}\right]$ Donc

$$
\|x(t)\|_{H} \leqslant c_{1}+c_{2} \frac{z}{\sqrt{n}}, \quad t \in\left[0, n^{-1}\right],
$$

ou $c_{1}$ et $c_{2}$ sont des constantes On deduit que

$$
\|x\|_{L^{2}\left(0{ }^{\prime}{ }^{1} H\right)}^{2}=\|x\|_{1 / n}^{2}\left(c_{1}+c_{2} \frac{z}{\sqrt{n}}\right)^{2} \frac{1}{n}
$$

et alors

$$
\begin{aligned}
& E^{2}\left\|\tilde{L}\left(x+e_{n}\right)\right\|_{1 / n}^{2}+\omega^{2} \geqslant E^{2}[z \sqrt{n}(\|\tilde{L}\|-\varepsilon)+ \\
& \left.-\|\tilde{L}\|\left(c_{1}+c_{2} \frac{z}{\sqrt{n}}\right)\right]^{2} \frac{1}{n}+\omega^{2} \geqslant z^{2}+ \\
& +\left\{\omega^{2}-\left[1-E^{2}(\|\tilde{L}\|-\varepsilon)^{2}\right] z^{2}-\frac{2 z}{\sqrt{n}}\left(c_{1}+c_{2} \frac{z}{\sqrt{n}}\right)\|\tilde{L}\|^{2} E^{2}\right\}
\end{aligned}
$$

Pour tout $M>0$ on prend $z \geqslant M, \in$ tellement petit que

$$
1-E^{2}(\|\tilde{L}\|-\varepsilon)^{2} z^{2} \leqslant \frac{\omega^{2}}{3}
$$

(ce quı est loisıble parce que $E\|\tilde{L}\| \geqslant 1$ ) et enfin $n$ suffisamment grand pour avoir $2 z n^{-1 / 2} E^{2}\|\tilde{L}\|^{2}\left(c_{1}+c_{2} \frac{z}{\sqrt{n}}\right) \leqslant \frac{\omega^{2}}{3}$ Alors

et donc $e_{n} \in \Gamma(\tilde{L})$

$$
\begin{gathered}
E^{2}\left\|\tilde{L}\left(x+e_{n}\right)\right\|_{\text {१ิ }}^{2}+\omega^{2} \geqslant E^{2}\left\|\tilde{L}\left(x+e_{n}\right)\right\|_{1 / n}^{2}+\omega^{2} \\
z^{2}+\frac{\omega^{2}}{3}>z^{2}=\left\|e_{n}\right\|_{y}^{2}
\end{gathered}
$$

Remarque - L'hypothese $\omega^{2}>0$ est essentielle lorsque $E\|\tilde{L}\|=1$

Demonstration de la Proposition 3 - Il suffit de demontrer qu'll existe $z \geqslant 1$ tel que, avec $L=z \tilde{L}$, on ait $z=1+\alpha_{T}(L)$, ce qui revient a resoudre le systeme

$$
\left\{\begin{array}{l}
\alpha=z-1 \\
\alpha=\varphi(z) \equiv \alpha_{T}(z \tilde{L})
\end{array}\right.
$$

Or $\varphi$ est strictement positive et, puisque $x_{0} \neq 0$, depend continuement de $z$ parce que $x(L)$ depend contınuement de $L$ (Kato [6]) De plus on a la majoration

$$
\varphi(z) \leqslant g_{T}(z) \equiv E\|\tilde{L}\| z\left\{1+\frac{\omega^{2}}{E^{2}\|\widetilde{L}\|^{2} z^{2}\|x(z \widetilde{L})\|_{T}^{2}}\right\}^{1 / 2}
$$


On démontrera dans un instant le

Lemme 3. $-z^{2}\|x(z \tilde{L})\|_{T}^{2} \rightarrow+\infty$ lorsque $z \rightarrow+\infty$.

Alors le graphe de $g_{T}$ rencontre la droite $\alpha=z-1$, parce que $E\|\tilde{L}\|<1$.

Puisque le graphe de $\varphi$ est connexe on voit qu'il existe au moins une solution de (64).

Démonstration du Lemme 3. $-x(z \tilde{L})$ est solution sur $[0, T]$ de

$$
\frac{\mathrm{d} x}{\mathrm{~d} t}=(A+z B L) x, x(0)=x_{0} \in D(A)
$$

et donc elle satisfait à l'égalité de l'énergie

$$
|x(t)|_{H}^{2}-\left|x_{0}\right|_{H}^{2}=2 Q(t)=2 \int_{0}^{t}((A+z B \tilde{L}) x(s), \quad x(s))_{V^{\prime}, V} \mathrm{~d} s .
$$

Si $b=\|B \tilde{L}\|_{\mathcal{L}(\boldsymbol{H})}$ alors $\left\|e^{(A+z B \tilde{L}) s}\right\| \leqslant \mathrm{e}^{z b s}$ parce que $A$ est de contraction. On déduit :

$$
|2 Q(t)| \leqslant 2 \int_{0}^{t} \mathrm{e}^{2 z b s}\left|x_{0}\right|\left[\left|A x_{0}\right|+z b\left|x_{0}\right|\right] \mathrm{d} s
$$

et donc

$$
|x(t)|^{2} \geqslant\left|x_{0}\right|^{2}\left\{1-\left[\frac{\left|A x_{0}\right|}{z b\left|x_{0}\right|}+1\right]\left(\mathrm{e}^{2 b z t}-1\right)\right\} .
$$

Si $z$ est suffisamment grand pour avoir $\left|A x_{0}\right|<z b\left|x_{0}\right|$, on a

$$
|x(t)|^{2} \geqslant\left|x_{0}\right|^{2}\left(3-2 \mathrm{e}^{2 b z t}\right) \geqslant \frac{1}{2}\left|x_{0}\right|^{2}
$$

si $t \leqslant t_{1}=(2 b z)^{-1} \ln 5 / 4$. Donc

$$
\|x(z \tilde{L})\|_{T}^{2} \geqslant \frac{1}{2} \int_{0}^{t_{1}}\left|x_{0}\right|^{2} \mathrm{~d} s=\frac{\ln 5 / 4}{4 b z}
$$

ce qui entraîne le Lemme. donc

$$
g_{T}(z) \leqslant E\|\tilde{L}\| z\left(1+C z^{-1}\right)^{1 / 2}
$$

avec $C$ indépendante de $T$. Alors les $\alpha_{T}(L)$ solutions de (64) sont bornées uniformément par rapport à $T$. 
On n'arrive pas à démontrer que l'ensemble des $\tilde{L}$ telles que $E\|\tilde{L}\|<1$ est contenu dans l'image de $\mu$, lorsque $T=+\infty$. Mais dans la démonstration du Théorème 3 on peut remplacer l'utilisation de (48) par le résultat de la proposition suivante.

Proposition 4. - Soit $E\|\tilde{L}\|<1$. Alors il existe $L \in \mathcal{H}$ telle que

$$
\sup _{e \in \Gamma(\tilde{L})} K(\tilde{L}, e) \geqslant \max _{e \in \mathcal{E}(L x(L))} \emptyset(L, e) .
$$

Démonstration. - Soient

$$
\begin{aligned}
& K_{T}(\tilde{L}, e)=\|\tilde{L}[X(e, \tilde{L})+e]\|_{T}^{2}+\|X(e, \tilde{L})+e\|_{r}^{2}, \\
& \Gamma_{T}(\tilde{L})=\left\{e \in L^{2}(0, T ; Y)\|\|\left\|_{T}^{2} \leqslant E^{2}\right\| \tilde{L}(X(e, \tilde{L})+e) \|_{T}^{2}+\omega^{2}\right\},
\end{aligned}
$$

$\emptyset_{T}(L, e), \Sigma_{T}(L x(L))$ définis analoguement.

On considère une suite $T_{n} \rightarrow+\infty$; pour tout $T_{n}$ il existe, par la proposition 3, $L_{n}$ telle que $\tilde{L}=\mu_{T_{n}}\left(L_{n}\right)$. Soit $\alpha_{n}=\alpha_{T_{n}}\left(L_{n}\right)$, uniformément borné (remarque précédente). Soit $\alpha_{m}$ une sous-suite convergente $\alpha_{m} \rightarrow \alpha^{*}$. Si $z_{m}=1+\alpha_{m}$, on a $z_{m} \rightarrow z_{\infty}=1+\alpha^{*}$ et $L_{m}=z_{m} \tilde{L} \rightarrow z_{\infty} \tilde{L}=L_{\infty}$ dans $\mathfrak{L}(H, U)$.

Si $L_{\infty} \notin \mathcal{M}$, alors $\forall M>0$ on peut trouver $T$ tel que

$$
\emptyset_{T}\left(L_{\infty}, \alpha^{*} x\left(L_{\infty}\right)\right)>M
$$

et pour $m$ suffisamment grand on a aussi $\emptyset_{T}\left(L_{m}, \alpha_{m} x\left(L_{m}\right)\right)>M$ et $T_{m}>T$. On définit $e_{m} \in \mathcal{Y}$ par

$$
e_{m}(t)=\left\{\begin{array}{lll}
\alpha_{m} x\left(L_{m}\right)(t) & \text { si } & t \leqslant T \\
0 & \text { si } & t>T
\end{array}\right.
$$

alors puisque $\tilde{L}=\left(1+\alpha_{m}\right)^{-1} L_{m}$ on a $e_{m} \in \Gamma(\tilde{L})$ et

$$
\begin{aligned}
K\left(\tilde{L}, e_{m}\right) & \geqslant K_{T_{m}}\left(\tilde{L}, \alpha_{m} x\left(L_{m}\right)\right)=\emptyset_{T_{m}}\left(L_{m}, \alpha_{m} x\left(L_{m}\right)\right) \geqslant \\
& \geqslant \emptyset_{T}\left(L_{m}, \alpha_{m} x\left(L_{m}\right)\right)>M ;
\end{aligned}
$$

donc $\sup K(\tilde{L}, e)=+\infty$.

$e \in \Gamma(\tilde{L})$

Si au contraire $L_{\infty} \in \mathcal{H}$, alors $\alpha_{\infty}=\alpha\left(L_{\infty}\right)$ est bien défini (mais pas nécessairement égal à $\alpha^{*}$ ) et pour tout $T>0$ et $\varepsilon>0$ on peut trouver $m$ tellement grand que $T_{m}>T$ et

$$
\emptyset_{T}\left(L_{m}, \alpha_{m} x\left(L_{m}\right)\right) \geqslant \emptyset_{T}\left(L_{\infty}, \alpha_{\infty} x\left(L_{\infty}\right)\right)-\varepsilon .
$$

$\mathrm{n}^{\circ}$ mars 1976. 
Puisque $\emptyset_{T}\left(L_{m}, \alpha_{m} x\left(L_{m}\right)\right)$ est croissant avec $T$ et $\check{L}=\mu_{T_{m}}\left(L_{m}\right)$ on trouve :

$$
\begin{aligned}
K\left(\tilde{L}, e_{m}\right) & \geqslant K_{T_{m}}\left(L, \alpha_{m} x\left(L_{m}\right)\right)=\emptyset_{T_{m}}\left(L_{m}, \alpha_{m} x\left(L_{m}\right)\right) \geqslant \\
& \geqslant \emptyset_{T}\left(L_{m}, \alpha_{m} x\left(L_{m}\right)\right) \geqslant \emptyset_{T}\left(L_{\infty}, \alpha_{\infty} x\left(L_{\infty}\right)\right)-\varepsilon .
\end{aligned}
$$

Mais, $T$ et $\varepsilon$ étant arbitraires, on a

$$
\sup _{e \in \Gamma(\tilde{L})} K(\tilde{L}, e) \geqslant \emptyset\left(L_{\infty}, \alpha_{\infty} x\left(L_{\infty}\right)\right)=\max _{e \in \Sigma\left(L_{\infty} x\left(L_{\infty}\right)\right)} \emptyset\left(L_{\infty}, e\right)
$$

parce que $\emptyset_{T}\left(L_{\infty}, \alpha_{\infty} x\left(L_{\infty}\right)\right)$ converge par $L_{\infty} \in \mathcal{M}$.

c.q.f.d.

On peut maintenant conclure la démonstration du théorème 3 : par la proposition 2 il suffit de considérer les $\tilde{L}$ telles que $E\|\tilde{L}\|<1$; mais alors la proposition 4 et $(51)$ montrent que (48) est vraie.

c.q.f.d.

\section{VARIATION DE LA FONCTION COUT ET EQUIVALENCE DU CONTROLE DE MIN-MAX EN BOUCLE OUVERTE ET FERMEE}

On considère la fonction $J_{\gamma^{*}}(u, e)$, définie par (23) au n.ro 3. Puisque $\gamma^{*}>1$, elle a un point selle unique $\left(u^{*}, e^{*}\right)$ et

$$
e^{*}(t)=\frac{1}{\gamma^{*}-1}\left(W u^{*}+f\right)(t)=\frac{1}{\gamma^{*}-1} x\left(u^{*}\right)(t)
$$

Le contrôle $u^{*}(t)$ est aussi fonction de l'état $x(t)$ (n.ro 4, (42)) :

$$
u^{*}(t)=L^{*} x(t)=-\frac{1}{1+\gamma^{*} E^{2}} B^{*} P_{\gamma^{*}} x(t),
$$

où $P_{\gamma^{*}}$ est l'opérateur de Riccati pour le problème 3 sur $[0,+\infty[$.

On applique maintenant les feedbacks $L^{*}$ et $\left(\gamma^{*}-1\right)^{-1}$ sur le nouveau système. On considère des perturbations $\tilde{u}$ et $\tilde{e}$ comme dans la fig. 3 .

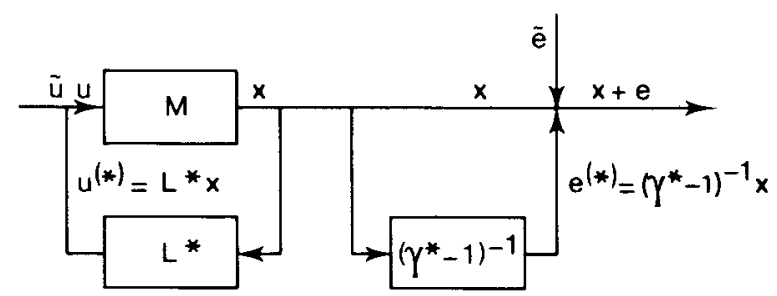

Figure 3

Revue Françaıse d'Automatıque, Informatıque et Recherche Opératıonnelle 
Le nouveau système est décrit par les équations :

$$
\begin{aligned}
& \frac{\mathrm{d} x}{\mathrm{~d} t}=A x+B L^{*} x+B \tilde{u}, \quad x(0)=x_{0} \\
& e=\left(\gamma^{*}-1\right)^{-1} x+\tilde{e}=e^{(*)}+\tilde{e} ;
\end{aligned}
$$

l'entrée totale $u$ est

$$
u=L^{*} x+\widetilde{u}=u^{(*)}+\tilde{u} .
$$

On remarque que si $\tilde{u} \neq 0$ alors $u^{(*)} \neq u^{*}$ et $e^{(*)} \neq e^{*}$, parce que l'état $x$ dépend de $\tilde{u}$.

On veut calculer les variations de $J_{\gamma^{*}}$ en fonction des perturbations $\tilde{u}$ et $\widetilde{e}$.

Lemme 4. - Pour tout $u \in \mathcal{U}$ et $e \in \mathcal{Y}$ on a :

$$
\begin{aligned}
J_{\gamma^{*}}\left(u, e^{(*)}+\tilde{e}\right) & =\left(1+\gamma^{*} E^{2}\right)\|u\|_{\mathscr{U}}^{2}+\frac{\gamma^{*}}{\gamma^{*}-1}\|W u+f\|_{\mathfrak{Y}}^{2}+ \\
& -\left(\gamma^{*}-1\right)\|\tilde{e}\|_{\mathfrak{y}}^{2}+\gamma^{*} \omega^{2} .
\end{aligned}
$$

Démonstration. - Puisque $e^{(*)}=\left(\gamma^{*}-1\right)^{-1}(W u+f)$, la formule de Taylor entraîne (78).

$$
\text { c.q.f.d. }
$$

La formule (78) montre que le maximum de $J_{\gamma^{*}}$ pour tout $u$ fixé est atteint en $e^{(*)}$.

Soit

$$
\psi(u)=\left(1+\gamma^{*} E^{2}\right)\|u\|_{u}^{2}+\frac{\gamma^{*}}{\gamma^{*}-1}\|W u+f\|_{y}^{2}
$$

on est intéressé à la fonction $\tilde{u} \rightarrow \psi\left(u^{(*)}+\widetilde{u}\right)$.

Pour l'analyse suivante il faut un résultat général sur les opérateurs de Riccati.

Avec les mêmes hypothèses du n.ro 1 on considère le système

$$
\frac{\mathrm{d} x}{\mathrm{~d} t}=A x+B u, \quad x(0)=x_{0}, \quad y=C x, \quad t \in[0, T]
$$

et la fonction coût quadratique

$$
\emptyset(u)=(R u, u)_{ฯ 1}+(Q y, y)_{\mathfrak{y}},
$$

avec $R \in \mathfrak{L}(U)$ et $Q \in \mathfrak{L}(Y)$.

La minimisation de $\emptyset$ sur $\mathcal{~} U$, avec $y$ donné par $(80)$, conduit à l'équation de Riccati.

$$
\frac{\mathrm{d} P}{\mathrm{~d} t}+P A+A^{*} P-P B R^{-1} B^{*} P+C^{*} Q C=0, \quad P(T)=0
$$

$\mathrm{n}^{\circ}$ mars 1976. 
et la trajectoire optimale $\hat{x}$ est solution de

$$
\frac{\mathrm{d} \hat{x}}{\mathrm{~d} t}=A \hat{x}-B R^{-1} B^{*} P \hat{x}, \quad \hat{x}(0)=x_{0} ;
$$

on a donc $\hat{y}=C \hat{x}$ et le contrôle optimal est

$$
\hat{u}=-R^{-1} B^{*} P \hat{x} .
$$

On considère le système modifié par le feedback (84) et la commande supplémentaire $v$ :

$$
\frac{\mathrm{d} x}{\mathrm{~d} t}=A x-B R^{-1} B^{*} P x+B v, \quad x(0)=x_{0} .
$$

Soit $W_{P}=\Omega_{P} B$ l'opérateur d'évolution associé à $(85)$ et soit $f_{P}$ la sortie libre de (85), c'est-à-dire la solution de (85) avec $v=0$.

On définit la fonctionnelle

$$
F(v)=\left\|v-R^{-1} B^{*} P\left(W_{P} v+f_{P}\right)\right\|_{R}^{2}+\left\|C\left(W_{P} v+f_{P}\right)\right\|_{Q}^{2},
$$

où $\|u\|_{R}^{2}=(R u, u)_{\text {ฯ }}$ et $\|y\|_{Q}^{2}=(Q y, y)_{y}$.

Théorème 4. - On a $F(0)=\emptyset(u)$,

$$
F(v)=F(0)+\|v\|_{R}^{2}\left({ }^{1}\right)
$$

l'opérateur $\Omega_{P}$ satisfait à l'équation

$$
-\Omega_{P}^{*} P-P \Omega_{P}+\Omega_{P}^{*} P B R^{-1} B^{*} P \Omega_{P}+\Omega_{P}^{*} C^{*} Q C \Omega_{P}=0
$$

Démonstration. - Lorsque $v=0(85)$ se réduit à $(83)$, donc $F(0)=\emptyset(u)$. De plus $F$ est minimum en 0 , sinon $F\left(v_{0}\right)<F(0)$ entraîne $\emptyset\left(u_{0}\right)<\emptyset(\hat{u})$, avec $u_{0}=v_{0}-R^{-1} . B^{*} P\left(W_{P} v_{0}+f_{P}\right)$, ce qui est impossible.

Par la formule de Taylor on a alors :

$$
F(v)=F(0)+\left\|C W_{P} v\right\|_{Q}^{2}+\left\|\left(1-R^{-1} B^{*} P W_{P}\right) v\right\|_{R}^{2}=F(0)+(\Lambda v, v)_{\Upsilon 1},
$$

avec

$$
\Lambda=W_{P}^{*} C^{*} Q C W_{P}+\left(1-R^{-1} B^{*} P W_{P}\right)^{*} R\left(1-R^{-1} B^{*} P W_{P}\right) .
$$

Il faut démontrer que $\Lambda=R$; mais $W_{P}=\Omega_{P} B$ et $W_{P}^{*}=B^{*} \Omega_{P}^{*}$, donc il suffit de démontrer (88).

Soit $D_{t}=\frac{d}{\mathrm{~d} t} ;$ l'équation de Riccati (82) peut s'écrire sous la forme

$$
\begin{aligned}
-C^{*} Q C+P\left(D_{t}-A+B R^{-1} B^{*} P\right)-\left(D_{t}+A^{*}\right. & \left.-P B R^{-1} B^{*}\right) P+ \\
& -P B R^{-1} B^{*} P=0
\end{aligned}
$$

(1) Voir dans une situation analogue Faurre [5]. 
Mais on a $\left(D_{t}-A+B R^{-1} B^{*} P\right) \Omega_{P} x=x$ et aussi

$$
\Omega_{P}^{*}\left(D_{t}+A^{*}-P B R^{-1} B^{*}\right) x=x \quad \text { si } \quad x(T)=0 \text {; }
$$

donc si on multiplie (91) à gauche par $\Omega_{P}^{*}$ et à droite par $\Omega_{P}$ on trouve (88).

Du théorème 4 on déduit

$$
\psi\left(u^{(*)}+\tilde{u}\right)=\psi\left(u^{*}\right)+\left(1+\gamma^{*} B^{2}\right)\|\tilde{u}\|_{\mathfrak{u}}^{2} .
$$

On a donc démontré la

Proposition 4. - La variation de $J_{\gamma^{*}}$ pour les perturbations $\tilde{u}$ et $\tilde{e}((75)$, (76), (77) et fig. 3) est donnée par

$$
\begin{aligned}
J_{\gamma^{*}}\left(u^{(*)}+\tilde{u}, e^{(*)}+\tilde{e}\right)=J_{\gamma^{*}}\left(u^{*}, e^{*}\right) & + \\
& +\left(1+\gamma^{*} E^{2}\right)\|\tilde{u}\|^{2}-\left(\gamma^{*}-1\right)\|\tilde{e}\|^{2}
\end{aligned}
$$

On va finalement démontrer le

Théorème 5. - Soit $\mathscr{H}_{\gamma}$ définie par

$$
\mathcal{H}_{\gamma^{*}}=\frac{1}{1+\gamma^{*} E^{2}} P_{\gamma^{*}} B B^{*} P_{\gamma^{*}}-\frac{\gamma^{*}}{\gamma^{*}-1},
$$

alors si $\mathcal{H}_{\gamma^{*}} \leqslant 0$ on a

$$
\min _{\tilde{L} \in[(H, U)} \max _{e \in \Gamma(\tilde{L})} K(\tilde{L}, e)=\min _{u \in \mathcal{U}} \max _{e \in \Sigma(u)} J(u, e)
$$

et le minimum est atteint en

$$
\tilde{L}^{*}=\mu\left(L^{*}\right)=\frac{\gamma^{*}-1}{\gamma^{*}} L^{*}
$$

Démonstration. - Pour tout $e \in \Gamma\left(\tilde{L}^{*}\right)$ on considère l'équation

$$
\frac{\mathrm{d} x}{\mathrm{~d} t}=A x+B \tilde{L}^{*}(x+e), \quad x(0)=x_{0}
$$

et l'on décompose $e$ suivant

$$
e=e^{(*)}+\tilde{e}=\left(\gamma^{*}-1\right)^{-1} x+\tilde{e}
$$

On a alors

$$
\begin{aligned}
\tilde{L}^{*}(x+e) & =\frac{\gamma^{*}-1}{\gamma^{*}} L^{*}\left[\left(1+\frac{1}{\gamma^{*}-1}\right) x+\tilde{e}\right]= \\
& =L^{*} x+\frac{\gamma^{*}-1}{\gamma^{*}} L^{*} \tilde{e}=u^{(*)}+\tilde{u} .
\end{aligned}
$$

$n^{\circ}$ mars 1976. 
On utilise la proposition 4 avec $\tilde{u}=\frac{\gamma^{*}-1}{\gamma^{*}} L^{*} \tilde{e}$ et l'on trouve pour tout $e \in \Gamma\left(\widetilde{L}^{*}\right)$

$$
\begin{aligned}
K\left(\tilde{L}^{*}, e\right) & =J\left(\tilde{L}^{*}(x+e), e\right) \leqslant J_{\gamma^{*}}\left(\tilde{L}^{*}(x+e), e\right)= \\
& =J_{\gamma^{*}}\left(u^{(*)}+\tilde{u}, e^{(*)}+\tilde{e}\right)=J_{\gamma^{*}}\left(u^{*}, e^{*}\right)+ \\
& \left.+\left[\frac{\gamma^{*}-1}{\gamma^{*}}\right]^{2} \mathcal{H}_{\gamma^{*}} e, e\right) \leqslant J_{\gamma^{*}}\left(u^{*}, e^{*}\right)= \\
& =J\left(u^{*}, e^{*}\right),
\end{aligned}
$$

avec egalite sı $\tilde{e}=0$ Donc

$$
\max _{e \in \Gamma\left(\tilde{L}^{*}\right)} K\left(\tilde{L}^{*}, e\right)=\min _{u \in \mathcal{U}} \max _{e \in \Sigma(u)} J(u, e),
$$

ce quı avec (48) entraîne (95)

\section{BIBLIOGRAPHIE}

[1] Bensoussan A, Saddle points of convex-concave functionals with applications to linear quadratic differential games in Differential Games and Related Topics, Proceedings of the International Summer School, Varenna 1970, North-Holland 1971

[2] DANSKIN J M, The theory of max-min, Springer, Berlin, 1967

[3] Donati $F$ et Milanese M, System identification ulth approximated models, 2nd IFAC Symposium, Prague, '70

[4] Ekeland I et Teman R, Analyse convexe et problemes variationnels, Dunod, Paris 1974

[5] FAURRE P, Linear differential games with completely optimal strategies, IFAC, Prague, 1966

[6] Kato T, Perturbatıon theory for linear operators, Sprınger, Berlın, 1966

[7] LemAIRE B, Saddle point problems in partial differential equations and applications to linear quadratıc differential games, to appear

[8] Lions J L, Contrôle optımal de systeme gouvernes par des equatıons aux derivees partielles, Dunod, Paris, 1968

[9] Menga G et Milanese M , Control of systems in presence of uncertainty in norm, Preprints of VI International Summer School on Electronics and Automation, Herceg Novi, Yougoslavia 1971

[10] MiLANESE M , Identification of uniformly approximating models of systems, Ricerche di Automatica, Vol II, n 2, 1971

[11] Milanese $M$ et Negro A, Uniform approximation of systems A Banach space approach, Journal of Optimization Theory and Application, Vol 11, n 5, 1973

[12] Negro A , Application of the Banach-Stemhaus Theorem to best approximation of systems, Bollettıno della U M I (4), 7, 1973, pp 176-185

[13] SChwartz L, Cours d analyse, Hermann, Parıs, 1967 